\title{
Liquid self-balancing device effects on flexible rotor stability
}

\author{
Leonardo Urbiola-Soto ${ }^{\mathrm{a}, *}$ and Marcelo Lopez-Parra ${ }^{\mathrm{b}}$ \\ ${ }^{a}$ Department of Mechanical Engineering, Universidad Nacional Autonoma de Mexico (UNAM), Campus \\ Juriquilla, Queretaro, Mexico \\ ${ }^{\mathrm{b}}$ Department of Mechanical Engineering, Universidad Nacional Autonoma de Mexico (UNAM), Campus \\ Juriquilla, Queretaro, Mexico
}

Received 26 April 2012

Revised 7 June 2012

\begin{abstract}
Nearly a century ago, the liquid self-balancing device was first introduced by M. LeBlanc for passive balancing of turbine rotors. Although of common use in many types or rotating machines nowadays, little information is available on the unbalance response and stability characteristics of this device. Experimental fluid flow visualization evidences that radial and traverse circulatory waves arise due to the interaction of the fluid backward rotation and the baffle boards within the self-balancer annular cavity. The otherwise destabilizing force induced by trapped fluids in hollow rotors, becomes a stabilizing mechanism when the cavity is equipped with adequate baffle boards. Further experiments using Particle Image Velocimetry (PIV) enable to assess the active fluid mass fraction to be one-third of the total fluid mass. An analytical model is introduced to study the effects of the active fluid mass fraction on a flexible rotor supported by flexible supports excited by bwo different destabilizing mechanisms; rotor internal friction damping and aerodynamic cross-coupling. It is found that the fluid radial and traverse forces contribute to the balancing action and to improve the rotor stability, respectively.
\end{abstract}

Keywords: Flexible rotor, liquid balance ring, rotordynamic stability

\section{Nomenclature}

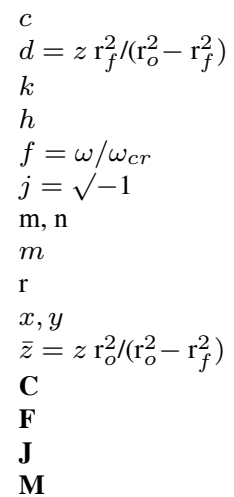

Damping, $\mathrm{N} \cdot \mathrm{s} / \mathrm{m}$

Distance from $\mathbf{C}$ to $\mathbf{F}, \mathrm{m}$

Stiffness, N/m

Balance ring height, $\mathrm{m}$

Frequency ratio, dimensionless

Imaginary unit, dimensionless

Circumferential and lateral modes of vibration

Mass, $\mathrm{kg}$

radius, $\mathrm{m}$

Cartesian coordinates, $\mathrm{m}$

Distance from $\mathbf{O}$ to $\mathbf{F}, \mathrm{m}$

Disk center

Fluid center of gravity

Journal center

Disk center of gravity

\footnotetext{
${ }^{*}$ Corresponding author: Leonardo Urbiola-Soto, Adjunct Professor, Department of Mechanical Engineering, Universidad Nacional Autonoma de Mexico (UNAM), Campus Juriquilla, Queretaro 76230, Mexico. E-mail: leourbiola@gmail.com.
} 


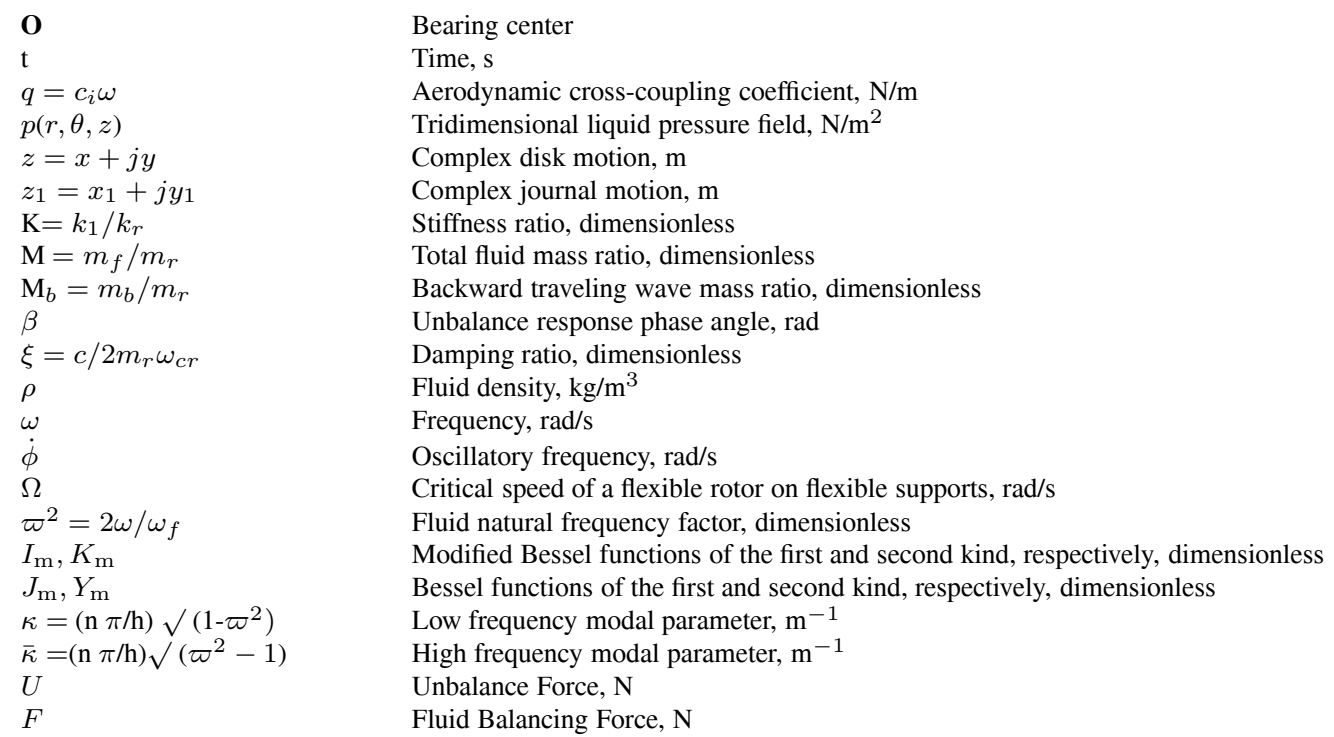

\section{Subscripts}

$\begin{array}{ll}1 & \text { Bearing property } \\ b & \text { Fluid traveling backwards property } \\ c r & \text { Rotor critical speed on rigid supports, rad/s } \\ \mathrm{d} & \text { Whirl frequency, rad/s } \\ e & \text { Effective property } \\ f & \text { Fluid property } \\ \mathrm{i} & \text { Rotor internal property } \\ i & \text { Inner } \\ m & \text { Maximum value } \\ o & \text { Outer } \\ o p & \text { Optimum value } \\ r & \text { Rotor property } \\ f w d & \text { Forward traveling wave } \\ b w d & \text { Backward traveling wave }\end{array}$

\section{Introduction}

There has been considerable work in the area of the dynamics of rotors with internal damping, particularly with regard to internal friction arising from micro-slip at shrink-fit interconnections of built-up rotors. Newkirk [1] pointed that internal rotor damping created by shrink fits of impellers and spacers is a predominant cause of whirl instability. Kimball [2] by means of deriving the equations of motion showed that the internal friction force tends to put the shaft motion in an ever-increasing spiral path. Gunter [3] developed a linear rotordynamic model in which internal friction was modeled as a cross-coupled force. He demonstrated that if external damping is added, the threshold speed could be greatly improved. He also showed that foundation asymmetry without foundation damping can cause a large increase of the onset speed of instability. Black [4] investigated a variety of models (viscous, Coulomb and hysteretic) for internal friction and differentiated between various models in reference to their ability to accurately predict the onset of instability. Lund [5] also investigated internal friction models, specifically due to micro-slip at axial splines and shrink fit joints. Srinivasan [6] showed that at some values of low interference fit, the system became unstable at high speeds, while no instability was noted for tighter fits. Damping for the low interference fit was higher than for the high interference. The aforementioned is due to the fact that when the fit is tight, slipping at the interference between the disk and the shaft is reduced. In most cases, the whirl instability can be suppressed with hardware fixes such as changing the bearings to softer supports with asymmetric stiffness, adding more damping in 


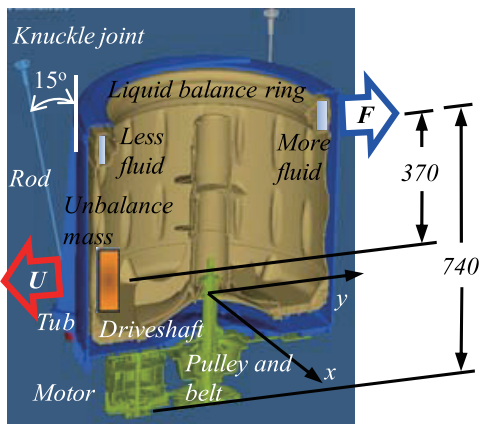

(a)

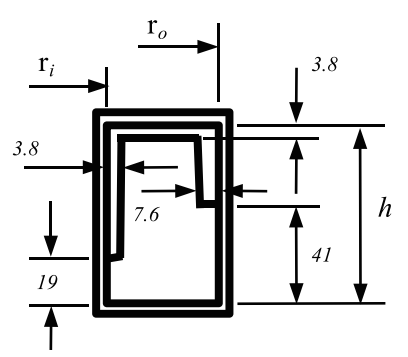

(b)

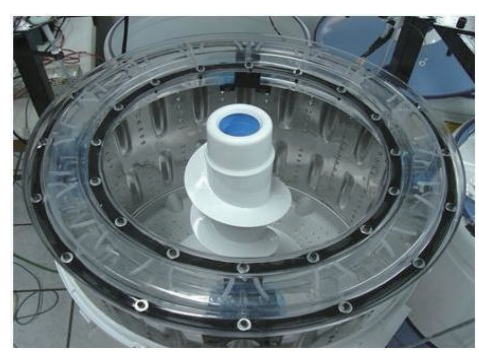

(c)

Fig. 1. Test rig; (a) main dimensions; (b) transparent balance ring; (c) cross section, dimensions in mm.

the bearings or tightening the interference fits. This paper proposes an alternative mechanism for improving rotor stability by using a LeBlanc balancer.

The LeBlanc balancer is basically a hollow ring equipped with a number of pockets formed by radial walls and partially filled with liquid. The limited information on this liquid balance ring made public can be found on some patents and a few technical papers and thesis work. Back in 1914, LeBlanc [7] first introduced a passive dynamic balancing device for turbine rotors consisting of an annular cavity partially filled with a liquid of high viscosity. However, this kind of device has not received much attention for practical use in turbomachiney since then. It has extensively been demonstrated that when a flexible rotor is partially filled with liquid, the motion is unstable over some operating range [8-12]. The extent of this operating range depends on various system parameters such as rotor damping, stiffness, fluid viscosity, the amount of fluid present, etc. However, as will be shown later, when the cavity filled with liquid is provided with a number of radial baffle boards, the unstable behavior changes dramatically, helping the rotating system achieve higher stable operating speeds.

Liquid may be in the interior of a rotor by design, as in the case of centrifuges, certain blade cooling systems, or in balance ring devices employed in washing machines. Also, liquids can be contained in rotating bodies by accident, as in the case of restarting turbines with trapped condensate inside. This paper shows experiments that indicate that the otherwise unstable mechanism consisting of trapped fluid in hollow rotors may become a stabilizing means if the cavity is equipped with radial baffles yielding; a) a fluid distribution within the cavity, and b) a raised fluid modes of vibration such that a fluid backward traveling wave occurs contributing to increase the threshold speed of instability of a flexible rotor. Then, the LeBlanc balancer can be used as a stability device to overcome destabilizing mechanism such as rotor internal damping and aero-dynamic cross-coupling.

A large scale balance ring equipped with a number of eight baffles boards and partially filled with a brine solution at a ratio of 0.5 is employed. Fluid flow visualization and Particle Image Velocimetry (PIV) are used to understand the fluid-solid interaction. Later, the active mass fraction of the fluid is quantified as one-third of the total fluid mass, which travels backwards to the rigid body rotation. This active fluid mass fraction is introduced as a damping mechanism into a 4 degrees of freedom model (DOF) of the flexible rotor on flexible supports. Linear stability analysis is carried out to determine the onset of unstable dynamic response using this model. Industrial turbomachinery rotors are included as examples. Balance ring blades and fluid inertia are found to play an important role in the onset of instability.

\section{Experimental apparatus}

The image on Fig. 1(a) represents a cut-away view of a modern washing machine, which comprises a tub that contains a rotating basket, the later capped by a liquid balance ring. An unbalance mass is placed inside the basket to simulate its unbalanced condition. The tub has in its bottom a centered opening through which a vertical driveshaft passes, which is coupled to the bottom of the basket in order to transmit rotational movement to it. The opening in the tub is sealed using a lip seal. The driveshaft is propelled by a motor, which is mounted below the tub. The axis of the rotor of such motor is parallel to the driveshaft. These components are coupled through a pulley and 


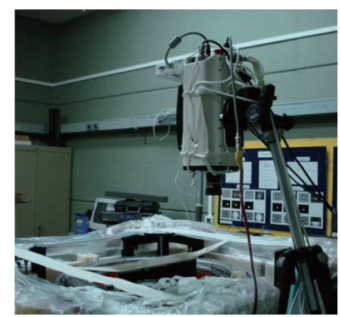

(a)

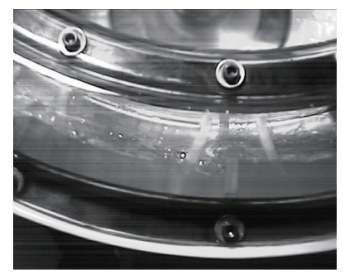

(b)

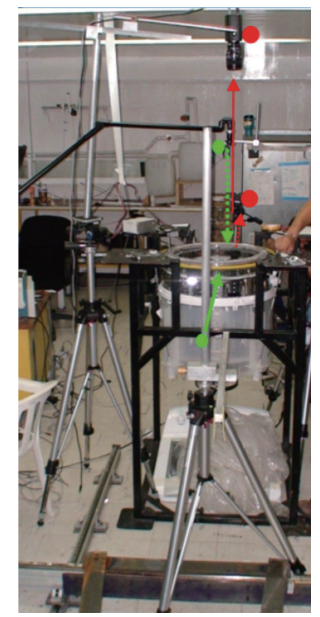

(c)

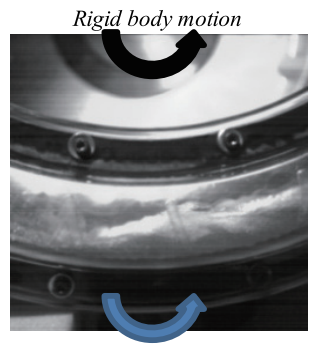

Fluid attached to rigid body

(a)

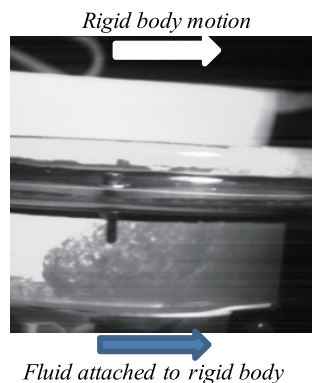

(b)

Fig. 2. Fluid flow visualization; (a) high-speed camera top view, (b) fluid flow attached to outer wall at $15.7 \mathrm{rad} / \mathrm{s}(150 \mathrm{rpm})$, and (c) PIV experimental array.

Fig. 3. Fluid bulk-flow in a hollow ring with no baffles; (a) top view, and (b) side view.

a belt. The tub is supported by a suspension consisting of a set of four rods that in their lower part account with a spring and an air damper. Such suspension assemblies are attached at the bottom to the tub and at the top are supported by the machine enclosing cabinet structure (not shown). The first natural frequency without fluid in the balance ring is located at $240 \mathrm{rpm}$, while the natural frequency with fluid in the ring cavity relocated at $200 \mathrm{rpm}$. The test rig is capable of running at $1000 \mathrm{rpm}$. Figure 1(b) shows the balance ring under study consisting of a Thermoformed Polyethylene Terephthalate (PET) ring assembled by two parts. The bottom part houses the baffles, which are inserted in machined guiding grooves. The upper part works as a top cover bolted to the bottom portion. The balance ring assembly is rigidly mounted on top of the rotating drum through a set of brackets. Clearances are provided with the inner and outer wall, and also with the top cover. This helps achieve higher order fluid modes of vibration. The balance ring employed mainly consists of an annular cavity of $3.709 \mathrm{E}-03 \mathrm{~m}^{3}$ equipped with a number of eight radial baffles equiangularly spaced, which dimensions are illustrated in Fig. 1(c). Sodium chloride with a density of $1300 \mathrm{~kg} / \mathrm{m}^{3}$ was employed to fill the cavity.

A high-speed camera at 1000 frames/s and a set of white light sources of Xenon lamps type were used for direct fluid flow visualization. A laser beam was orthogonally oriented with respect to the direction of the observing camera by using an articulated arm system. PIV is an optical method of fluid visualization, it is used to obtain instantaneous velocity measurements and related properties in fluids. The fluid is seeded with tracer particles which, for the purposes of PIV, are generally assumed to follow the flow dynamics. The particle concentration must be such that it is possible to identify individual particles in an image. It is the motion of these seeded particles that is used to calculate velocity information of the flow being studied. A detailed discussion on PIV and its historical development is provided by Adrian [13]. The PIV technique employed permitted the visualization and quantification of bidimensional velocity fields of the fluid through the seeding of particles of alumina whose diameter ranges from $5 \mathrm{y} 50 \mu \mathrm{m}$. Figure 2 shows the experimental array. The flow was lightened through a laser beam of Yttrium Aluminum Garnet doped with Neodymium (Nd:YAG). The light spread by the particles reaches a Charge Coupled Device (CCD) image sensor camera, which in turn generates consecutive images of the flow. Since the laser beam is of very high frequency nature (pulses of $5 \mathrm{~ns}$ ), it is possible to "freeze" two consecutive fluid motion frames and from them, build the velocity vectors. The plant view of the balance ring was made on an area of $90 \times 65 \mathrm{~mm}$, while for the lateral view an area of $240 \times 170 \mathrm{~mm}$ was employed.

\section{Fluid flow visualization and PIV results}

As reported by Urbiola-Soto and Lopez-Parra [14], experiments were performed in the test rig with an unbalanced rotor. This was not unstable in the absence of fluid in the ring cavity, but it underwent large vibrations due to the 


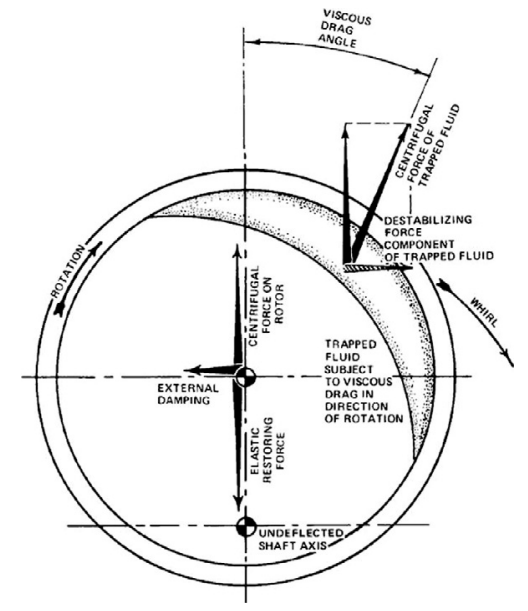

Fig. 4. Whirl due to fluid trapped in rotor, from [15].

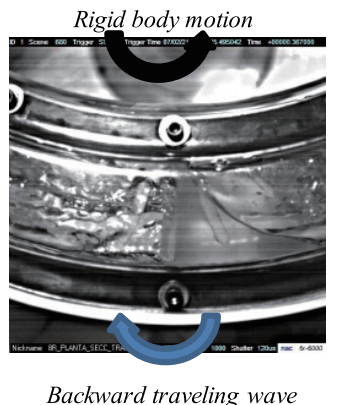

(a)

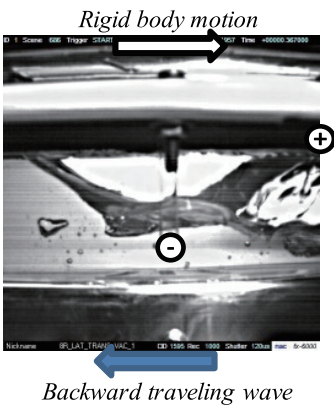

(b)
Fig. 5. Backward traveling wave, (a) top view, and (b) side view, (+) and $(-)$ indicate a crest and valley, respectively.

synchronous unbalance response. When tested with fluid filling the ring cavity at ratios of 0.5 and 0.8 , the unbalance response was synchronous and greatly reduced due to the balancing action of the fluid. However, regardless of the fill ratio, the removal of baffles inside the ring rendered a partially wetted cavity as shown on Fig. 3. A strong unstable vibration occurred at speeds as low as $200 \mathrm{rpm}$, dwelling in the system natural frequency in the first 2 to 3 seconds after the start up, while the drum rotor barely completed 6 to 10 turns. This almost leaded to destruction of the rig and disabled experimental vibration measurements. However, video recording was managed to be taken to observe the rotor excursions, which never reached steady-state. This behavior is similar to trapped fluids (e.g., oil from bearing sumps, steam condensate, etc.) in the internal cavity of high-speed hollow rotors [15].

As explained by [15], the fluid does not remain in simple radial orientation. The spinning surface of the cavity drags the fluid (which has some finite viscosity) in the direction of rotation. This fluid shear stress results in a transverse force in the direction of rotation. This force is called follower force and is the fundamental condition for instability as shown on Fig. 4. On the contrary, the addition of baffles showed that the fluid is not stationary relative to the rigid body, see Fig. 5. Furthermore, the baffles increased the natural frequency of the fluid and induced a complex fluid mode of vibration. The experimental rig was always stable at speeds as high as $1000 \mathrm{rpm}$ once the baffles were put in place inside the ring.

The stability of the test rig once the cavity of the ring was equipped with baffles to resemble a LeBlanc balancer is following discussed. The rotor passed from being quite unstable at speeds as low as $200 \mathrm{rpm}$ to reach stable rotating speeds up to $1000 \mathrm{rpm}$. This remarkable increment in the onset of instability is due solely to the presence of the baffles in the cavity as confirmed in the experiments presented. In other words, the instability due to trapped fluids in hollow rotors typically shows plastered liquid on one side of the cavity causing fluid shear stress and a destabilizing follower force in the direction of rotation [15]. Whereas, when baffles are provided in the cavity, the fluid mode of vibration increases of order and the liquid distributes around the ring wetting the whole cavity, thereby suppressing the trapped fluid instability. This contributes to; 1) the well-known LeBlanc self-balancing effect, and 2) a backward traveling wave with a mass $m_{f}$ adding a damping effect. This added damping due to the backward traveling waves is evidenced by the phase lag in between the unbalance (heavy spot) and displacement vector (high sport), see Fig. 6. Should other destabilizing sources exist in the rotating system, such as rotor internal damping or aerodynamic cross-coupling for example, the added damping by the active fluid mass $\left(m_{f}\right)$ may contribute to enhance the rotor stability. Figure 6 shows the fluid distribution relative to the unbalance mass location at steadystate. Note that an angle of $30^{\circ}$ exists between the heavy spot location and the thinnest fluid zone. Owing to the fact that, the fluid center of mass is aligned with the displacement vector, then there is a complementary angle of $150^{\circ}$ between the heavy spot location and the fluid center of mass. In other words, the unbalance force is not exactly opposed by the fluid balancing force, thus showing that there is a phase lag due to the fluid damping effect.

As shown on Fig. 1(c) the radial baffles placed in the cavity account with gaps with the inner and outer walls, this enables a backward traveling fluid wave that describes a swirl at low speeds, Fig. 5(b). Establishing an analogy with 


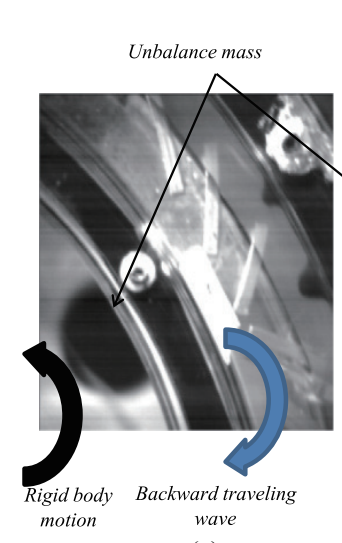

(a)

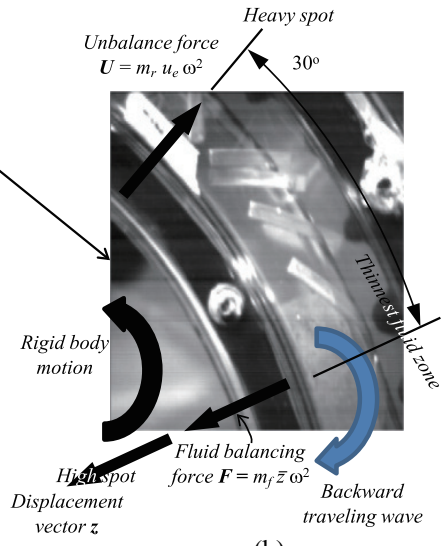

(b)
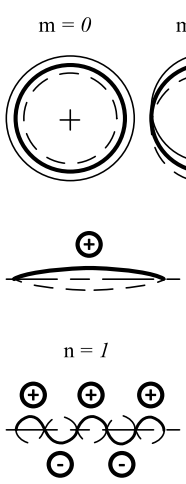

$\mathrm{n}=5$
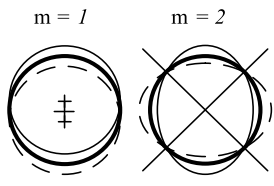
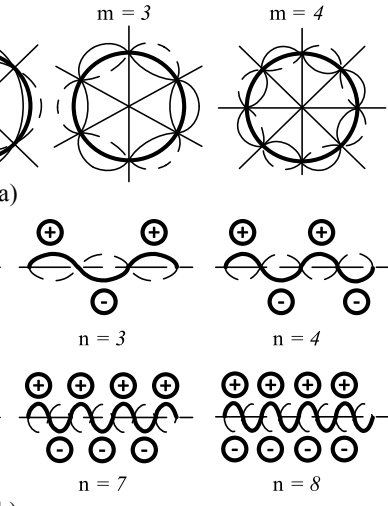

(b)

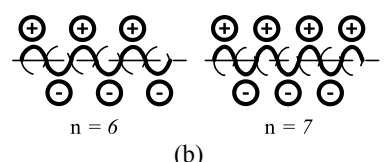

Fig. 7. Modes of vibration; (a) circumferential modes of vibration of a thin wall ring, and (b) lateral mode shapes of a string.

6. Fluid distribution relative to unbalance mass location steady-state; (a) unbalance mass location; (b) relative location of thinnest fluid zone to unbalance mass location.

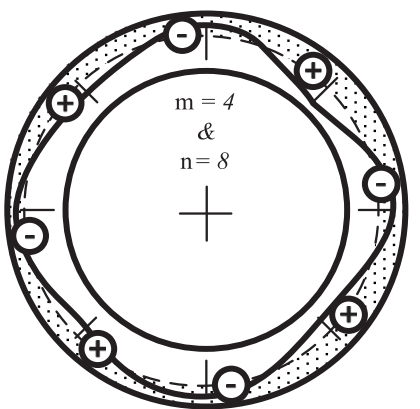

(a)

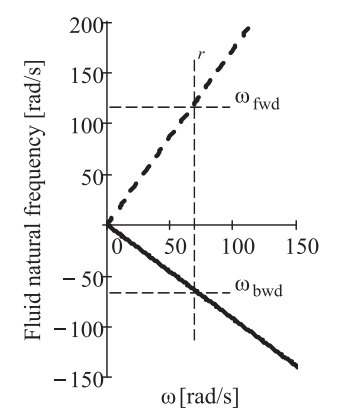

(b)

Fig. 8. Complex 3D wave; (a) side view with crest and valley, and (b) coupled vibrating modes; $\mathrm{m}=4$ and $\mathrm{n}=8$.
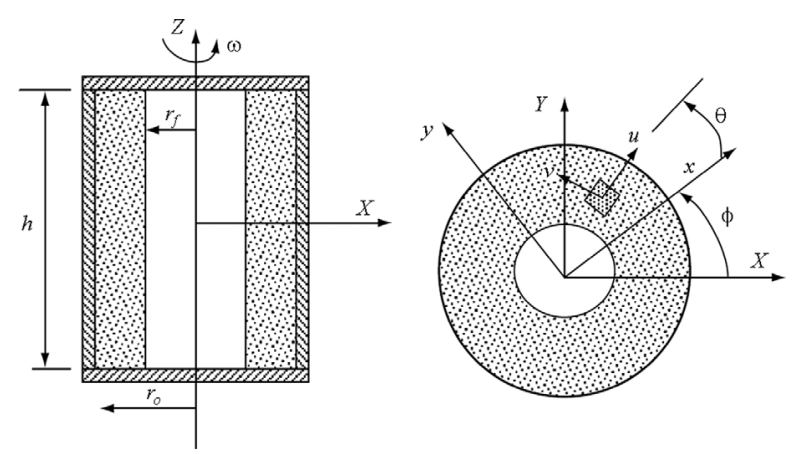

Fig. 9. Rotating tank partially filled with liquid, from [10].

the circumferential modes of vibration of a ring with m-modes, it can be seen from Fig. 7 [16] that the fourth mode is comparable to the fluid waves induced by the baffle-fluid interaction in the circumferential direction. Furthermore, a comparison with the lateral modes of vibration of a string for the n-modes leads to ascertain that the eighth lateral mode of vibration makes a parallel with the axial wave induced by the baffle-fluid interaction. Coupling the fourth circumferntial mode of vibration of a ring with the eighth lateral mode of a string, a complex 3D wave shown schematically on Fig. 8(a) is obtained.

On the other hand, in a symmetrical tank spinning about its z-symmetry axis, the theory shows, that the liquid pressure field $p(\mathrm{r}, \theta, \mathrm{z}) e^{(-j \dot{\phi} \mathrm{t})}=0$ corresponding to an oscillation frequency of $\dot{\phi}$ is governed by

$$
\frac{\partial p}{\partial \mathrm{r}^{2}}+\frac{\partial^{2} p}{\mathrm{r}^{2} \partial \theta^{2}}+\left(1-\frac{4 \omega^{2}}{\dot{\phi}^{2}}\right) \frac{\partial^{2} p}{\partial \mathrm{z}^{2}}=0
$$

When the oscillation frequency $\dot{\phi}>2 \omega$, this differential equation is of elliptic form and the oscillations are the free surface sloshing oscillations mentioned above. But when $\dot{\phi}<2 \omega$, the equation is of hyperbolic nature and the oscillations are inertial waves [10]. No general theory is available for the problem under discussion, but approximate analytical models indicate that even for this nonaxisymmetric geometry all the inertial wave resonances occur in the frequency range between 0 and $2 \omega$, and the most prominent resonances have frequencies less than $\omega$. Miles and Troesch [10] considered the three-dimensional oscillations of the rotating tank system partially filled with liquid shown in Fig. 9.

The motion in the rotating body is transmitted to the contacting liquid by shear stress; this suggests that waves are occurring in the fluid. Since the spin axis does not coincide with the angular momentum vector, the drum rotor 


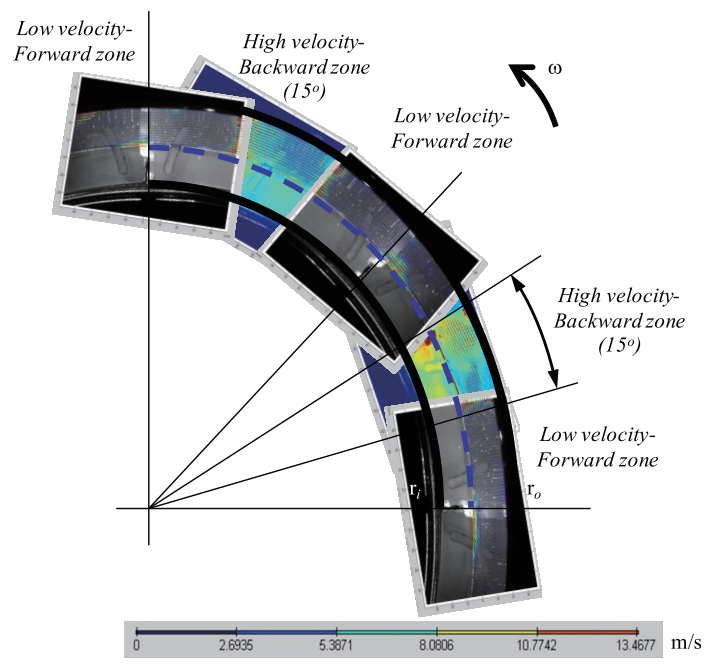

Fig. 10. Balance ring relative velocity map.

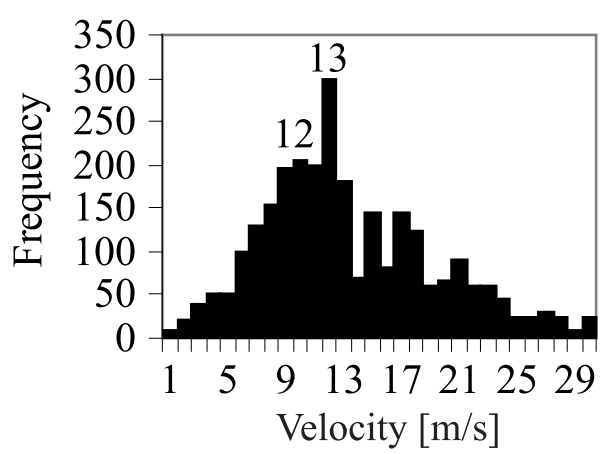

Fig. 11. Velocity histogram.

appears to oscillate about its transverse $x$ and $y$ axes. This oscillation or whirling causes the liquid in the ring to move relative to the rigid body. If the drum rotor spins about a major moment-of-inertia axis, the liquid motions tend to damp the whirling. The energy dissipated by the oscillatory motions is extremely large when the whirling motion excites the liquid into resonance. This motion does not relate to free-surface sloshing. According to Miles and Troesch [10], the motion occurs throughout the liquid interior and is thus called an internal wave or inertia oscillation. These researchers considered the three-dimensional oscillations of the rotating tank system partially filled wave fluid, and with its mth and nth excited natural frequencies. They showed that the disturbed motion of the fluid free-surface could not remain irrotational due to a vertical gradient of velocity that necessarily generates vorticity. They have outlined an approach for determining the slosh frequencies in spinning cylinders partially filled with liquid. The natural frequency of the free-surface can be obtanined from numerical solution of Eq. (2a) for the fluid natural frequency factor when $\varpi^{2}<1$, similarly Eq. (2b) is solved for the fluid natural frequency factor when $\varpi^{2}>1$.

Solution of the determinants renders the pair of fluid natural frequencies as depicted on Fig. 8(b), one frequency is positive and greater than the rotor angular velocity (fast wave) and the other is negative, i.e. traveling backwards relative to the rigid body, and it is slower than the rotor speed (slow wave).

$$
\begin{aligned}
& \left|\begin{array}{ll}
\kappa \mathrm{r}_{o} I_{\mathrm{m}}^{\prime}\left(\kappa r_{o}\right)+\mathrm{m} \varpi I_{\mathrm{m}}\left(\kappa \mathrm{r}_{o}\right) & \kappa \mathrm{r}_{o} K_{\mathrm{m}}^{\prime}\left(\kappa \mathrm{r}_{o}\right)+\mathrm{m} \varpi K_{\mathrm{m}}\left(\kappa \mathrm{r}_{o}\right) \\
\varpi^{2} \kappa \mathrm{r}_{i} I_{\mathrm{m}}^{\prime}\left(\kappa \mathrm{r}_{i}\right) & \varpi^{2} \kappa r_{i} K_{m}^{\prime}\left(\kappa r_{i}\right) \\
+\left[4\left(1-\varpi^{2}\right)+\mathrm{m} \varpi^{3}\right] I_{\mathrm{m}}\left(\kappa \mathrm{r}_{i}\right) & +\left[4\left(1-\varpi^{2}\right)+\mathrm{m} \varpi^{3}\right] K_{\mathrm{m}}\left(\kappa \mathrm{r}_{i}\right)
\end{array}\right|=0 \\
& \left|\begin{array}{ll}
\bar{\kappa} \mathrm{r}_{o} J_{\mathrm{m}}^{\prime}\left(\bar{\kappa} \mathrm{r}_{o}\right)+\mathrm{m} \varpi J_{\mathrm{m}}\left(\bar{\kappa} \mathrm{r}_{o}\right) & \bar{\kappa} \mathrm{r}_{o} Y_{\mathrm{m}}^{\prime}\left(\kappa \mathrm{r}_{o}\right)+\mathrm{m} \varpi Y_{\mathrm{m}}\left(\bar{\kappa} \mathrm{r}_{o}\right) \\
\varpi^{2} \bar{\kappa} \mathrm{r}_{i} J_{\mathrm{m}}^{\prime}\left(\bar{\kappa} \mathrm{r}_{i}\right) & \varpi^{2} \bar{\kappa} r_{i} Y_{m}^{\prime}\left(\bar{\kappa} r_{i}\right) \\
+\left[4\left(1-\varpi^{2}\right)+\mathrm{m} \varpi^{3}\right] J_{\mathrm{m}}\left(\bar{\kappa} \mathrm{r}_{i}\right) & +\left[4\left(1-\varpi^{2}\right)+\mathrm{m} \varpi^{3}\right] Y_{\mathrm{m}}\left(\bar{\kappa} \mathrm{r}_{i}\right)
\end{array}\right|=0
\end{aligned}
$$

PIV and unbalanced response tests evidenced the theoretical fast wave not to exist as will be following discussed. Only the slow negative wave turned out to be of importance. The stability mechanism is due to this backward traveling wave present in the fluid, which acts as an added damping force. The fluid theoretical natural frequency was computed to be an average of $0.983 \mathrm{x} \omega_{r}$, i.e. very much synchronous with the running speed $\omega_{r}$. The fluid relative velocity map was built by superposition of PIV frames for different portions of the balance ring as illustrated on Fig. 10.

This fluid mass fraction induces a transverse force $90^{\circ}$ phase lagged with the solid body motion, thus behaving as a positive damping stabilizing effect. The effective mass $\left(m_{b}\right)$ traveling backwards is a fraction of the total fluid mass given by Eq. (3)

$$
15^{\circ} \times 8 \text { High Relative Velocity Zones }=120^{\circ}=\frac{1}{3} \text { Circumference }
$$



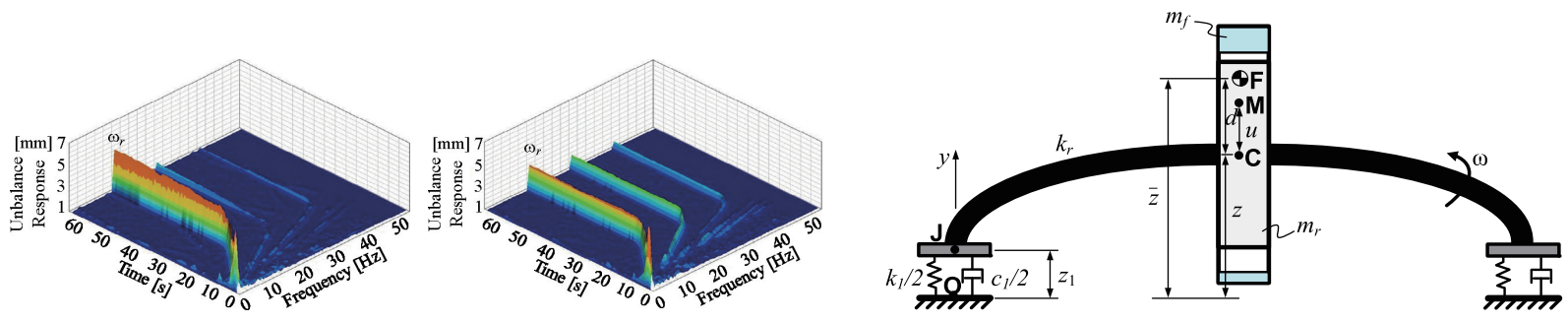

Fig. 12. Waterfall vibration plot of balance ring vibration; (a) $x$-axis, and (b) $y$-axis.

Fig. 13. Dynamic model of a flexible rotor with a LeBlanc Balancer.

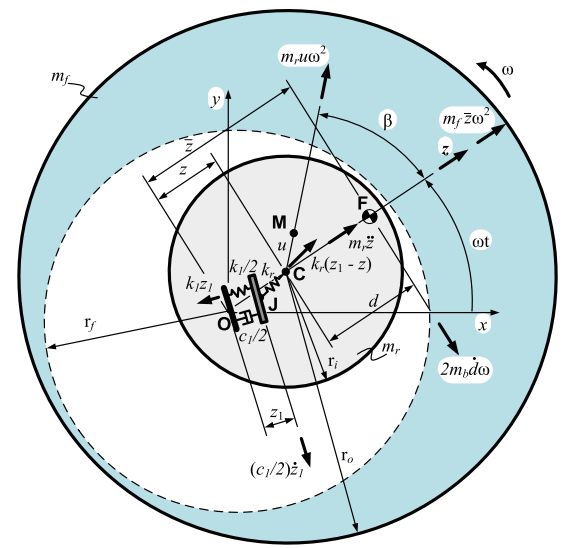

Fig. 14. Side view of flexible rotor with a LeBlanc Balancer.

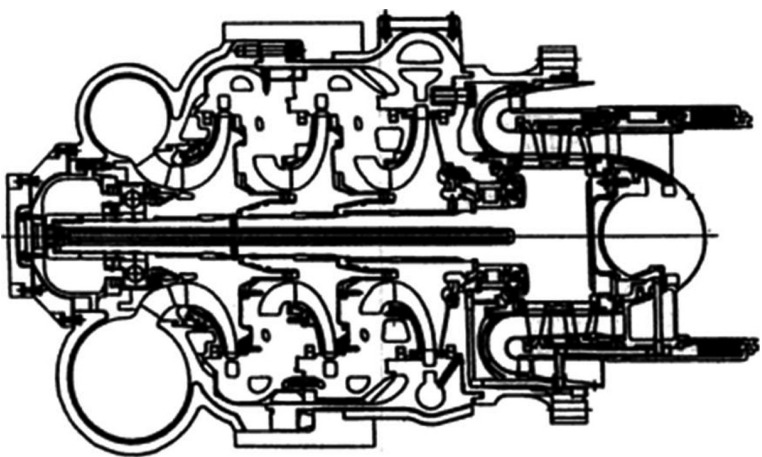

Fig. 15. Space Shuttle Main Engine-High Pressure Fuel Turbopump (SSME-HPFTP), from [5].

Therefore, the active "fluid damping mass" $\left(m_{b}\right)$ is one third of the total fluid mass $\left(m_{f}\right)$.

$$
m_{b}=0.33 m_{f}
$$

On the other hand, histogram of fluid velocity on Fig. 11 indicates the solid transverse velocity as $13 \mathrm{~m} / \mathrm{s}$, while the fluid average transverse velocity is $12 \mathrm{~m} / \mathrm{s}$. Furthermore, waterfall plots of balance ring vibration, shown on Fig. 12, evidence strong synchronous motion with $\omega_{r}$ and almost inexistent asynchronous activity.

\section{Unbalance response}

Figure 13 provides a 4-DOF dynamic model of a flexible rotor in flexible symmetric damped bearings and with a LeBlanc balancer.

It has been assumed that the bearings cross-coupled stiffness and damping coefficients are negligible as might be the case of tilting pad journal bearings and squeeze film dampers. Thus bearings are not considered to be a source of instability in this analysis. Furthermore, for the sake of simplicity, the bearings are assumed to be essentially identical and symmetric. Figure 14 provides a side view of the flexible rotor equipped with a LeBlanc balancer.

Then, for the flexible rotor, the differential equations of motion of the rotor are given by

$$
\begin{aligned}
& m_{r} \ddot{z}+k_{r}\left(z-z_{1}\right)+m_{f} \bar{z} \omega^{2}+2 m_{b} \dot{d} \omega=m_{r} u \omega^{2} e^{j \omega t} \\
& k_{1} z_{1}+k_{r}\left(z_{1}-z\right)+c_{1} \dot{z}_{1}=0
\end{aligned}
$$

Equations (5) and (6) are combined to give

$$
\ddot{z}+2 \omega_{c r} \xi_{e} \dot{z}+2 \mathbf{M}_{b} \dot{d} \omega+\Omega^{2} z+\mathbf{M} \bar{z} \omega^{2}=u \omega^{2} e^{j \omega t}
$$


Where $d$ and $\bar{z}$ are the distance of the fluid center of gravity to the disk and bearing center, respectively. They are obtained in Appendix 1, such that Eq. (7) becomes a second order linear differential equation with constant coefficients and can have a closed form solution as shown on Appendix 2. Note that a damping term $2 m_{b} \dot{d} \omega$ has been added, which can be thought mainly as viscous dissipation in the bulk-flow. In other words, this additional damping is due to the mass fraction moving backwards relative to the rigid body. The acceleration of the backward traveling wave, which mass is $m_{b}$, has no radial component and only possesses transverse or Coriolis acceleration given by $2 \dot{\boldsymbol{d}} \omega$. Therefore, its transverse force is defined by the term $2 m_{b} \dot{\boldsymbol{d}} \omega$, where the wave frequency is synchronous with $\omega$. Solution of Eq. (7) renders

$$
\begin{aligned}
z & =\frac{u}{\left[\left(\frac{\Omega^{2}}{\omega^{2}}-1+\frac{\rho \pi r_{o}^{2} h}{m_{r}}\right)^{2}+\left(2 \frac{\omega}{\omega_{c r}} \xi_{e}+2 \mathrm{M}_{b}\left(\frac{\mathrm{r}_{f}^{2}}{\mathrm{r}_{o}^{2}-\mathrm{r}_{f}^{2}}\right)\right)^{2}\right]^{1 / 2}} \\
z_{1} & =\frac{z}{\left[\left(\mathrm{~K}+1-\mathrm{Mf}^{2}\right)^{2}+\left(2 \xi_{1} \mathrm{f}\right)^{2}\right]^{1 / 2}}
\end{aligned}
$$

Where the critical speed of a flexible rotor on flexible supports is

$$
\Omega^{2}=\omega_{c r}^{2}\left[\frac{\mathrm{K}(\mathrm{K}+1)+\left(2 \mathrm{f} \xi_{1}\right)^{2}}{(\mathrm{~K}+1)^{2}+\left(2 \mathrm{f} \xi_{1}\right)^{2}}\right]
$$

The effective damping is given by

$$
\xi_{e}=\frac{\xi_{1}}{(\mathrm{~K}+1)^{2}+\left(2 \xi_{1} \mathrm{f}\right)^{2}}
$$

Gunter et al. [2] have shown that the effective damping can be maximized $\left(\xi_{\text {em }}\right)$ with respect to the bearing damping by finding the value of the bearing damping $\left(\xi_{1}\right)$ which satisfies $\partial \xi_{e} / \partial \xi_{1}=0$. Hence

$$
\xi_{\text {em }}=\frac{1}{4(\mathrm{~K}+1)}
$$

Substituting Eq. (12) into Eq. (11) and solving for the bearing damping $\left(\xi_{1}\right)$, the optimum bearing damping $\left(\xi_{1 o p}\right)$ is obtained as

$$
\xi_{1 o p}=\frac{\mathrm{K}+1}{2}
$$

The effects of aerodynamic cross-coupling and rotor internal damping will now be considered.

\section{Stability with optimum damping and a leblanc balancer}

Rotor bearing systems are frequently subjected to self-excited instability mechanisms associated with bearings, seals, aerodynamic effects, and internal rotor friction damping. For free damped vibrations with aerodynamic crosscoupling and optimum bearing damping, Eq. (7) becomes

$$
\ddot{z}+2 \omega_{c r} \xi_{e m} \dot{z}+2 \mathbf{M}_{b} \dot{d} \omega+\left(\Omega^{2}-j \mathbf{Q}\right) z+\mathbf{M} \bar{z} \omega^{2}=u \omega^{2} e^{j \omega t}
$$

Where $\xi_{\text {em }}$ is given by Eq. (12) and $\mathrm{Q}=q / m_{r}$

$$
\Omega_{o p}^{2}=\omega_{c r}^{2}\left[\frac{2 \mathrm{~K}+1}{2(\mathrm{~K}+1)}\right]
$$

With viscous internal friction damping Eq. (6) becomes

$$
\ddot{z}+2 \omega_{c r}\left(\xi_{\text {em }}+\xi_{\mathrm{i}}\right) \dot{z}+2 \mathbf{M}_{b} \dot{\boldsymbol{d}} \omega+\Omega^{2} z+\mathbf{M} \bar{z} \omega^{2}=u \omega^{2} e^{j \omega t}
$$

Assuming an exponential function of the form $z=\mathrm{z} e^{s t}$, the characteristic equation is of the form

$$
m_{r} s^{2}+\left[c_{e m}+c_{\mathrm{i}}+2 m_{b} \omega\left(\frac{\mathrm{r}_{f}^{2}}{\mathrm{r}_{o}^{2}-\mathrm{r}_{f}^{2}}\right)\right] s+\frac{\mathrm{K}(2 \mathrm{~K}+1)}{2(\mathrm{~K}+1)}+m_{f}\left(\frac{\mathrm{r}_{o}^{2}}{\mathrm{r}_{o}^{2}-\mathrm{r}_{f}^{2}}\right) \omega^{2}=0
$$


The values of $s$ satisfying Eq. (17) are the system eigenvalues. Since $s$ is complex, Eq. (17) represents a complex function. In order to be zero, both the real and imaginary parts must be simultaneously zero. Substituting $s=\lambda+j \omega_{d}$ into Eq. (17) and equating individually the real and imaginary part to zero results in a pair or polynomial with real coefficients, which need to be solved simultaneously. A rather simple solution for which the rotor becomes unstable can be found by substituting $\lambda=0$ to deliver

$$
\omega_{d_{o p}}=\left[\omega_{c r}^{2}\left(\frac{2 \mathrm{~K}+1}{2(\mathrm{~K}+1)}\right)+2 \omega^{2} \mathbf{M}_{b}\left(\frac{\mathrm{r}_{f}^{2}}{\mathrm{r}_{o}^{2}-\mathrm{r}_{f}^{2}}\right)\right]^{1 / 2}
$$

Whereas with viscous internal friction damping, the rotor will only be stable if the operating speed is

$$
\omega<\Omega_{o p}\left[1+\frac{1}{4(1+\mathrm{K}) \xi_{i}}+\mathrm{M}_{b} \frac{\mathrm{f}}{\xi_{\mathrm{i}}}\left(\frac{\mathrm{r}_{f}^{2}}{\mathrm{r}_{o}^{2}-\mathrm{r}_{f}^{2}}\right)\right]^{1 / 2}
$$

According to Eq. (18), when the rotor is unstable, it whirls at the undamped natural frequency plus the rising term depending on the traveling backwards mass of the LeBlanc balancer and its geometrical properties as well. On the other hand, Eq. (19) shows that for the rotor to be unstable due to internal friction damping, the rotational speed must exceed the undamped natural frequency plus a destabilizing term depending on the internal friction damping, plus a rising term depending on the traveling backward mass of the LeBlanc balancer and its geometrical properties.

While the maximum permissible aerodynamic cross-coupling with optimum damping is

$$
q_{m_{o p}}=2 m_{r} \omega_{c r} \omega_{d}\left[\xi_{e m}+\mathrm{M}_{b} \frac{\mathrm{f}}{2}\left(\frac{\mathrm{r}_{f}^{2}}{\mathrm{r}_{o}^{2}-\mathrm{r}_{f}^{2}}\right)\right]^{1 / 2}
$$

\section{Examples}

Industrial rotor cases will be taken from reference [17] to analyze the effect of the LeBlanc balancer to improve their stability. The internal damping for those rotors is unknown a priori. Some guessed values for the internal damping will be assumed for two conditions, namely loose and tight fit. The first example is a ten-stage centrifugal compressor, designated in the following as the "light-rotor". The unit is nearly symmetrical and the rotor is supported in two five-pad tilting pad bearings. A second example, considers the seven-stage centrifugal compressor, designated from now on as the "heavy rotor". This is a rotor mounted on very rigid pressure dam journal bearings with different stiffness values on the horizontal and vertical directions. The third example deals with an eight-stage centrifugal compressor supported on tilting pad journal bearings mounted in series with squeeze film dampers, where the stiffness values on the horizontal and vertical direction differ greatly from each other. The final example is the Space Shuttle Main Engine-High Pressure Fuel Turbopump (SSME-HPFTP). The HPFTP consists of a three-stage centrifugal pump section and a two-stage turbine section as shown in Fig. 15. Details of the pump configuration are given in reference [18]. The rotating assembly is supported in flexible mounted ball bearings and is acted on by aerodynamic cross-coupling forces in the turbine section. For the purpose of analyzing the stabilizing effect of the LeBlanc balancer, it will be assumed that little damping is provided to the rotor from the bearings, supports and seals. The supports will be considered symmetric and isotropic. Different bearing stiffness values will be considered.

A balance ring with dimensions $\mathrm{r}_{o}=0.102 \mathrm{~m} \mathrm{(4} \mathrm{in),} \mathrm{r}_{i}=0.076 \mathrm{~m} \mathrm{(3}$ in), $h=0.076 \mathrm{~m}$ (3 in), filled with a liquid density of $\rho=1300 \mathrm{~kg} / \mathrm{m}^{3}$ at ratio of 0.5 as recommend by [14] to maximize the balancing capabilities of the trapped fluid in the cavity. The fluid free-surface radius $r_{f}$ is obtained from geometrical analysis of Fig. 14 and given by Eq. (21).

$$
\mathrm{r}_{f}=\left[0.5\left(\mathrm{r}_{i}^{2}-\mathrm{r}_{o}^{2}\right)+\mathrm{r}_{o}^{2}\right]^{0.5}
$$

Table 1 summarizes the threshold speed of instability and maximum aerodynamic cross-coupling improvements for all the example rotors. 
Table 1

Summary of stability results of industrial example rotors employing a LeBlanc Balancer

\begin{tabular}{|c|c|c|c|c|c|c|c|c|c|c|c|c|c|c|c|}
\hline & & & & & & & & Rotor & Internal & Thresh & old spee & ed of ins & ability [rpn & & \\
\hline & & & & & & & & Dan & mping & & oose & & Tight & & \\
\hline Rotor & Direction & $\begin{array}{c}\text { Operating } \\
\text { speed } \\
{[\mathrm{rpm}]}\end{array}$ & $\begin{array}{l}\text { Balance } \\
\text { ring }\end{array}$ & $\mathrm{K}$ & $\begin{array}{c}\text { Modal } \\
\text { mass } \\
{[\mathrm{kg}]}\end{array}$ & $\begin{array}{c}\text { Critical } \\
\text { speed } \\
{[\mathrm{rpm}]}\end{array}$ & $\begin{array}{c}\text { Whirl } \\
\text { frequency } \\
\text { [cpm] }\end{array}$ & Loose & Tight & $\begin{array}{l}\omega_{\mathrm{d} o p} \\
{[\mathrm{rpm}]}\end{array}$ & $\begin{array}{c}\% \text { of } \\
\text { increase }\end{array}$ & $\begin{array}{r}\omega_{\mathrm{d} o p} \\
{[\mathrm{rpm}]}\end{array}$ & $\begin{array}{c}\% \text { of } \\
\text { increase } \\
\% \text { of } \\
\text { increase }\end{array}$ & $\begin{array}{c}\mathrm{q}_{\mathrm{m} o p} \\
{[\mathrm{~N} / \mathrm{cm}]}\end{array}$ & $\begin{array}{c}\% \text { of } \\
\text { increase }\end{array}$ \\
\hline & Horizontal & & $\begin{array}{l}\text { Without } \\
\text { With }\end{array}$ & 3.4 & & & $\begin{array}{l}3735 \\
3776\end{array}$ & 0.059 & 0.0313 & $\begin{array}{l}5148 \\
5377\end{array}$ & 4 & $\begin{array}{l}6398 \\
6794\end{array}$ & 6 & 21390 & 12 \\
\hline $\begin{array}{l}\text { Light } \\
\text { rotor }\end{array}$ & & $\begin{array}{l}\text { Above } \\
\text { critical }\end{array}$ & $\begin{array}{l}\text { With } \\
\text { Without }\end{array}$ & & 215 & 5100 & $\begin{array}{l}3 / 10 \\
4912\end{array}$ & & & $\begin{array}{l}53 / 1 \\
7928\end{array}$ & & $\begin{array}{c}0 / 94 \\
10597\end{array}$ & & $\begin{array}{l}24244 \\
42749\end{array}$ & \\
\hline & Vertical & & With & 5.9 & & & 4990 & 0.059 & 0.0313 & 8381 & 5 & 11382 & 7 & 48056 & 11 \\
\hline $\begin{array}{l}\text { Heavy } \\
\text { rotor }\end{array}$ & Symmetrical & $\begin{array}{l}\text { Above } \\
\text { critical }\end{array}$ & $\begin{array}{l}\text { Without } \\
\text { With }\end{array}$ & 29.2 & 2524 & 2540 & $\begin{array}{l}2519 \\
2522\end{array}$ & 0.059 & 0.0313 & $\begin{array}{l}2872 \\
2890\end{array}$ & 1 & $\begin{array}{l}3185 \\
3216\end{array}$ & 1 & $\begin{array}{l}29320 \\
30521\end{array}$ & 4 \\
\hline 8-stage & Horizontal & 000 & $\begin{array}{l}\text { Without } \\
\text { With }\end{array}$ & 10.2 & 306 & 382 & $\begin{array}{l}3735 \\
3776\end{array}$ & 0.059 & 0.0313 & $\begin{array}{l}5148 \\
5377\end{array}$ & 4 & $\begin{array}{l}6398 \\
6794\end{array}$ & 6 & $\begin{array}{l}21390 \\
24244\end{array}$ & 12 \\
\hline compressor & & 0.000 & $\begin{array}{l}\text { Without } \\
\text { With }\end{array}$ & 0.36 & 300 & 3821 & $\begin{array}{l}3038 \\
3088\end{array}$ & 0.059 & 0.03131 & $\begin{array}{l}12505 \\
12853\end{array}$ & 3 & $\begin{array}{l}20883 \\
21494\end{array}$ & 3 & $\begin{array}{l}143313 \\
147815\end{array}$ & 3 \\
\hline & & & $\begin{array}{l}\text { Without } \\
\text { With }\end{array}$ & 0.71 & & & $\begin{array}{l}13880 \\
15903\end{array}$ & 0.118 & 0.0626 & $\begin{array}{l}31076 \\
39450\end{array}$ & 21 & $\begin{array}{l}46295 \\
60290\end{array}$ & 23 & $\begin{array}{l}213187 \\
291923\end{array}$ & 27 \\
\hline $\begin{array}{l}\text { SSME- } \\
\text { HPFTP }\end{array}$ & Symmetrical & 28000 & $\begin{array}{l}\text { Without } \\
\text { With }\end{array}$ & 2.02 & 29 & 16500 & $\begin{array}{l}15072 \\
16954\end{array}$ & 0.118 & 0.0626 & $\begin{array}{l}25646 \\
32946\end{array}$ & 22 & $\begin{array}{l}35004 \\
47099\end{array}$ & 26 & $\begin{array}{l}131085 \\
198260\end{array}$ & 34 \\
\hline & & & $\begin{array}{l}\text { Without } \\
\text { With }\end{array}$ & 4.04 & & & $\begin{array}{l}15660 \\
17478\end{array}$ & 0.118 & 0.0626 & $\begin{array}{l}22243 \\
29051\end{array}$ & 23 & $\begin{array}{l}28069 \\
39293\end{array}$ & 29 & $\begin{array}{c}81610 \\
143471\end{array}$ & 43 \\
\hline
\end{tabular}

\section{Discussion}

The optimum bearing damping for all the rotors exemplified was determined and used as an input in the analysis. Despite of the optimization of the external damping and the relatively high internal rotor damping values employed, significant improvements in the threshold speed of instability and maximum aerodynamic cross-coupling were obtained for the light rotor case. However for the "heavy rotor" case, with the extremely stiff bearings, the flexible bearing critical speed is very close to the rigid bearing critical speed and even the external damping provided by the balance ring has little stabilizing effect since the bearing amplitudes are very small. The 8-stage centrifugal compressor with $\mathrm{K}=0.36$ in the vertical direction is another situation where the balance ring does not contribute much to the stability characteristics. In this case, the bearing supports flexibility is so large, that the external damping provided by the tilting pad journal bearings in series with the squeeze film dampers, is successful to provide enough damping to move forward the threshold speed of instability. The balance ring is of great help in the case of the SSME-HPFTP rotor, the internal rotor damping values used in this example are twice those for the other cases though. The apparent benefit is due to the high operating speed. This will need further experimental verification to ascertain whether the fluid behaves entirely as a solid at such extreme high-speed and confirm if the fluid backward traveling wave that provide the damping effect persists or vanishes.

The added mass of the balance ring was only $1.845 \mathrm{~kg}$, the dimensions of the ring are feasible and relatively easy to fit in the rotors design with some engineering work. This kind of devices can be particularly attractive for using in hollow rotors. Note that the balance ring will show even better stability improvements in rotors that by design do not have optimum external damping. The analysis can be further extended to consider asymmetric and orthotropic supports and other models for internal damping available in the literature.

\section{Conclusions}

Turbomachinery rotors are subject to a number of destabilizing mechanisms, such like trapped fluids, rotor internal damping, aerodynamic cross-coupling and others. It is widely known that trapped fluids in hollow rotors can lead to severe self-excited vibrations. However, if the cavity of a hollow rotor is equipped with radial baffles ressembling a LeBlanc balancer, the behavior of the fluid passes from being a destabilizing source to be an added stabilizing effect. This is due to the fluid's modes of vibration and the associated circulatory backward traveling wave that 
emerges adding damping to the system. This paper shows that this stabilizing mechanism can help alleviate the effects of two studied instabilities, namely; rotor internal damping and aerodynamic cross-coupling. Hollow rotors with fluid trapped inside, suspension segregation centrifuges, liquid-cooled gas turbines, spin-stabilized satellites, spinning rockets containing liquid fuel, home appliances such as washer machines, liquid-filled flywheel apparatus for motorcycles, automobiles, trailers and heavy vehicles tires self-balancing are just few examples of machines for which this kind of balancer is applicable not only to balance the rotating system but also to achieve much higher stable rotational speeds.

\section{Acknowledgments}

Thanks to Universidad Nacional Autónoma de México (UNAM) and the Consejo Nacional de Ciencia y Tecnología (Proyecto SEP-CONACYT-Ciencia Básica No. 83239) for granting support for presenting this paper.

\section{Appendix 1- derivation of distance from fluid C.G. to points $\mathrm{C}$ and $\mathrm{O}$}

Assuming the fluid center of gravity $\mathbf{F}$ to be coincident with its centroid, the distance of the former to $\mathbf{C}$ is obtained according to

$$
d=\frac{\Sigma C_{n} A_{n}}{\Sigma A_{n}}=\frac{(0) \pi r_{o}^{2}-(-z) \pi r_{f}^{2}}{\pi\left(r_{o}^{2}-r_{f}^{2}\right)}=z \frac{r_{f}^{2}}{r_{o}^{2}-r_{f}^{2}}
$$

Where $n$ stands for the $n$-th area $A_{n}$ which centroid is at a distance $C_{n}$ to the point $\mathrm{C}$.

Similarly, the distance of the fluid center of gravity to $\mathbf{O}$ is given by

$$
\bar{z}=\frac{\Sigma C_{n} A_{n}}{\Sigma A_{n}}=\frac{z_{i} \pi r_{o}^{2}-(0) \pi r_{f}^{2}}{\pi\left(r_{o}^{2}-r_{f}^{2}\right)}=z \frac{r_{o}^{2}}{r_{o}^{2}-r_{f}^{2}}
$$

Note that by employing $d$ and $\bar{z}$ as expressed above, Eqs (7) and (16) are put in terms solely of $z$ and its derivatives, such that easily solvable second order ordinary linear differential equations with constant coefficients are obtained. The terms $d$ and $\bar{z}$ are further useful substitution to simplify both, the unbalance response solution shown in Eq. (8), and the characteristic polynomial stated in Eq. (17).

\section{Appendix 2- simplification of equations of motion}

The differential equations of motion of a Jeffcott rotor mounted on symmetric flexible bearings are given by Eqs (5) and (6).

Dividing Eq. (5) by $m_{r}$ and since $\omega_{c r}^{2}=k_{r} / m_{r}$

$$
\ddot{z}+\omega_{c r}^{2}\left(z-z_{1}\right)+\mathbf{M} \bar{z} \omega^{2}+2 \mathbf{M}_{b} \dot{d} \omega=u \omega^{2} e^{j \omega t}
$$

Similarly, Eq. (6) renders Eq. (23).

$$
\frac{k_{1}}{m_{r}} z_{1}+\omega_{c r}^{2}\left(z_{1}-z\right)+\frac{c_{1}}{m_{r}} \dot{z}_{1}=0
$$

Since $\mathrm{K}=k_{1} / k_{r}$ and $\xi_{1}=c_{1} / 2 m_{r} \omega_{c r}$

$$
2 \omega_{c r} \xi_{1} \dot{z}_{1}+\mathrm{K} \omega_{c r}^{2} z_{1}+\omega_{c r}^{2}\left(z_{1}-z\right)=0
$$

Assuming particular function solutions of the form $z=z e^{j \omega t}$ and $z_{1}=z_{1} e^{j \omega t}$ and dividing by $\omega_{c r}^{2}$

$$
2 j \xi_{1} z_{1} \mathrm{f}+\mathrm{K} z_{1}+z_{1}-z=0
$$


Rearranging and solving for $z_{1}$ in terms of $z$.

$$
z_{1}=\frac{z}{\left[\mathrm{~K}+1+2 j \xi_{1} \mathrm{f}\right]}
$$

Multiplying by the complex conjugate in the numerator and denominator

$$
z_{1}=z \frac{\mathrm{K}+1-2 j \xi_{1} \mathrm{f}}{(\mathrm{K}+1)^{2}+\left(2 \xi_{1} \mathrm{f}\right)^{2}}
$$

Let $\mathrm{D}=(\mathrm{K}+1)^{2}+\left(2 \xi_{1} \mathrm{f}\right)^{2}$, then

$$
z_{1}=\frac{\mathrm{K}+1}{\mathrm{D}} z-\frac{2 \xi_{1}}{\mathrm{D} \omega_{c r}} \dot{z}
$$

Substitution of Eq. (24) into Eq. (22) delivers

$$
\begin{gathered}
\ddot{z}+\omega_{c r}^{2}\left[z-\frac{\mathrm{K}+1}{\mathrm{D}} z+\frac{2 \xi_{1}}{\mathrm{D} \omega_{c r}} \dot{z}\right]+\mathrm{M} \bar{z} \omega^{2}+2 \mathrm{M}_{b} \dot{d} \omega=u \omega^{2} e^{j \omega t} \ddot{z}+2 \omega_{c r} \frac{\xi_{1}}{\mathrm{D}} \dot{z} \\
+\omega_{c r}^{2}\left[1-\frac{\mathrm{K}+1}{\mathrm{D}}\right] z+\mathrm{M} \bar{z} \omega^{2}+2 \mathbf{M}_{b} \dot{d} \omega^{2}=u \omega^{2} e^{j \omega t}
\end{gathered}
$$

Which with further algebraic manipulation reduces to

$$
\ddot{z}+2 \omega_{c r} \xi_{e} \dot{z}+2 \mathbf{M}_{b} \dot{\boldsymbol{d}} \omega+\Omega^{2} z+\mathbf{M} \bar{z} \omega^{2}=u \omega^{2} e^{j \omega t}
$$

The latest has the form of Eq. (7).

\section{References}

[1] B.L. Newkirk, Shaft whipping, General Electric Review 27-3 (1924), 169-178.

[2] A.L. Kimball, Jr., Internal friction theory of shaft whirling, General Electric Review 27-4 (1924), $244-251$.

[3] E.J. Gunter, Dynamic stability of rotor-bearing systems, Nasa Technical Report, SP-113, 1966.

[4] H.F. Black, The stabilizing capacity of bearings for flexible rotors with hysteresis, Asme Journal of Engineering for Industry 2 (1976), 87-91.

[5] J.W. Lund, Destabilization of rotors from friction in internal joints with micro-slip, International Conference in Rotordynamics, JSME 1986, 487-491.

[6] A. Srinivasan, The influence of internal friction on rotordynamics instability, M.S. Thesis, Texas A\&M University, 2003.

[7] M. LeBlanc, Automatic balancer for rotating bodies, US Patent 1,159,052, 1914

[8] S.L. Hendricks and J.B. Morton, Stability of a rotor partially filled with a viscous incompressible fluid, ASME Paper 79-WA/APM-28, 48 (1979), 97-106.

[9] A.E. Hosoi and L. Mahadevan, Axial instability of a free-surface front in a partially filled horizontal rotating cylinder, Journal of Physics of Fluids 11-1 (1999), 97-106.

[10] J.W. Miles and B.A. Troesch, Surface oscillations of a rotating liquid, Journal of Applied Mechanics 28 (1961), $491-496$.

[11] Z. Changsheng, Experimental investigation into the effect of fluid viscosity on instability of an over-hung flexible rotor partially filled with liquid, ASME Journal of Vibrations and Acoustics 128 (2006), 1-10.

[12] E.F. Ehrich, The influence of trapped fluids on high speed rotor vibration, ASME Journal of Engineering for Industry 89 (1967), 806-812.

[13] R.J. Adrian, Twenty years of particle image velocimetry, Journal of Experiments in Fluids 39-2 (2005), 159-169.

[14] L. Urbiola-Soto and M. Lopez-Parra, Dynamic performance of the LeBlanc balancer for automatic washing machines, Asme Journal of Vibrations and Acoustics 133-4 (2011).

[15] F. Ehrich and D. Childs, Identification and avoidance of instabilities in high performance turbomachinery, Mechanical Engineering (1984), 66-79.

[16] S. Timoshenko, D.H. Young and W. Weaver, Jr., Vibrations Problems in Engineering, John Wiley \& Sons, 1974.

[17] L.E. Barret, E.J. Gunter and P.E. Allaire, Optimum bearing and support damping for unbalance response and stability of rotating machinery, ASME Journal of Engineering for Power 2 (1978), 89-94.

[18] D. Childs, Turbomachinery rotordynamics: phenomena, modeling, and analysis, John Wiley and Sons, New York, 1993. 

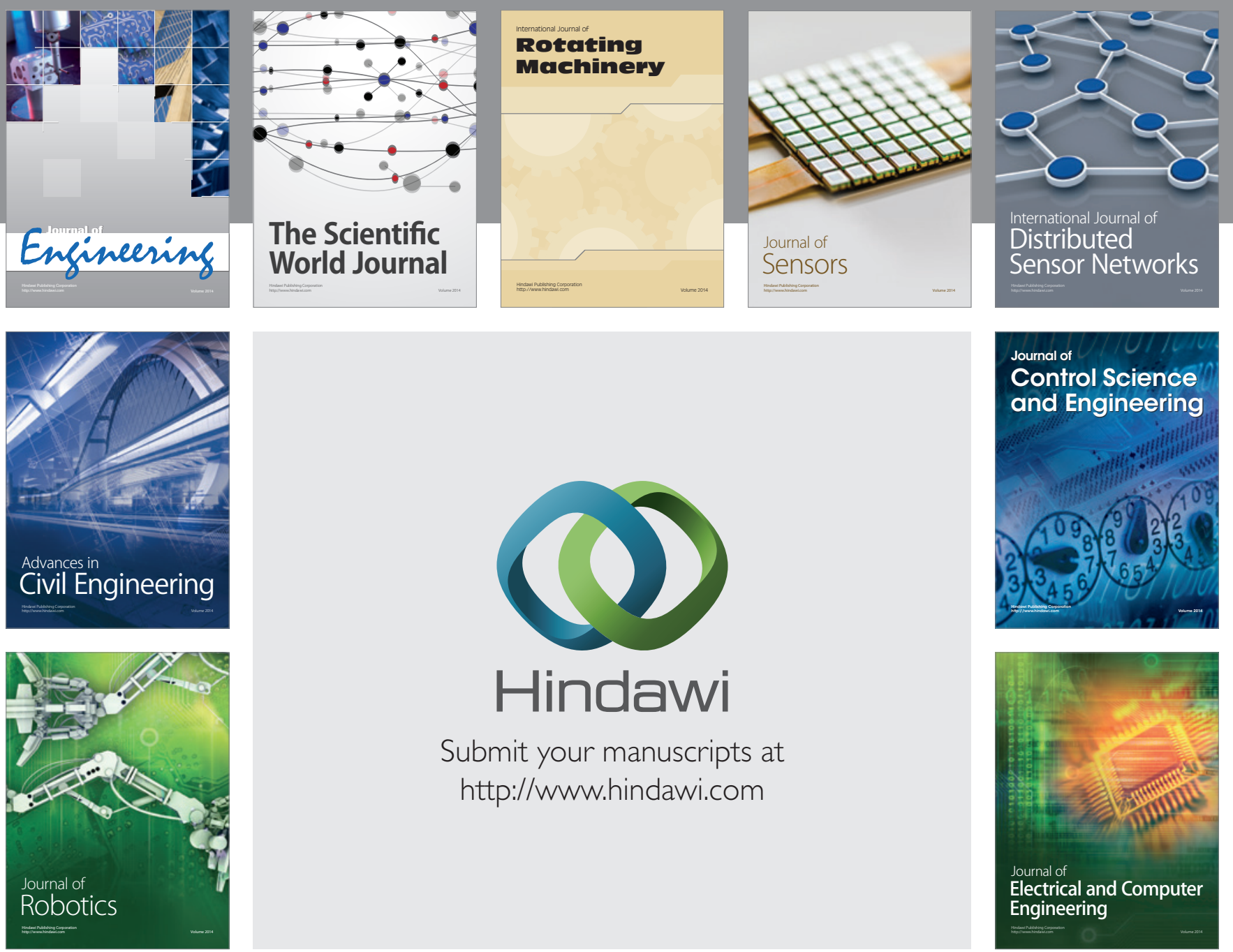

Submit your manuscripts at

http://www.hindawi.com
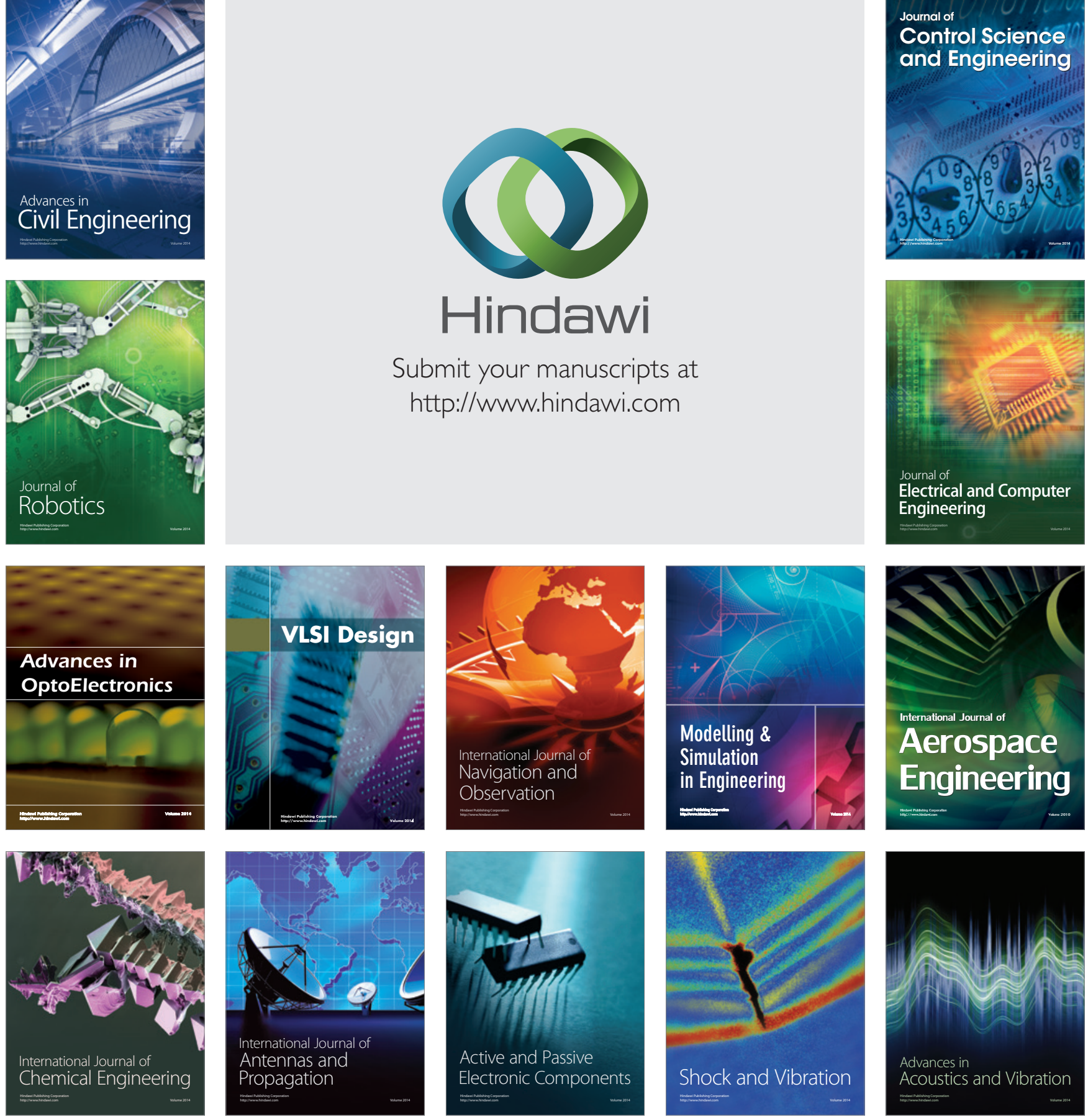